\title{
ON ASSOCIATIVE DIVISION ALGEBRAS ${ }^{1}$
}

\section{BY A. A. ALBERT}

1. Introduction. It seems highly appropriate to us that, in this address, ${ }^{2}$ we should present to you results which represent new progress in the structure theory of associative division algebras, a field untouched for nearly thirty years, and which provided the topic of our doctoral dissertation of 1928.

As in the paper already published, ${ }^{3}$ we shall study the structure of a central division algebra $\mathfrak{D}$, of odd prime degree $p$ over any field $\mathfrak{F}$ of characteristic $p$, which has the property that there exists a quadratic extension field $\Omega$ of $\mathfrak{F}$ such that the algebra $\mathfrak{D}_{0}=\mathfrak{D} \times \Omega$ is cyclic over $\Omega$. We shall obtain a simplified version of the JA condition that a cyclic algebra $\mathfrak{D}_{0}$, of degree $p$ over $\Re$, shall possess the factorization property $\mathfrak{D}_{0}=\mathfrak{D} \times \Re$. We shall also derive a new sufficient condition that such a $\mathfrak{D}$ shall be cyclic over $\mathfrak{F}$, and shall present a large class of our algebras $\mathfrak{D}_{0}$ which satisfy this condition.

These results still leave very much open the fundamental question of the existence of noncyclic division algebras of prime degree. However, they do show that we are still far from an end to the consideration of the algebraic aspects of the problem, and are not yet really ready for the computational attack proposed in JA.

2. The field $\mathfrak{N}$. The main ingredient of our study of factorizable algebras $\mathfrak{D}_{0}$ over $\Re$ is a certain inseparable field $\mathfrak{N}$. We let $\Re=\mathfrak{F}(u)$ be a quadratic extension of $\mathfrak{F}$ so that we can assume that $u^{2}=\mu$ in $\mathfrak{F}$. Then $\Omega$ has an automorphism $\gamma=\gamma_{1}+\gamma_{2} u \rightarrow \bar{\gamma}=\gamma_{1}+\gamma_{2} u$ for every $\gamma_{1}$ and $\gamma_{2}$ of $\mathfrak{F}$, and this conjugate operation has period two. Let $g$ be an element of $\Re$ and $\Re\left(y_{0}\right)$ be a splitting field of $\mathfrak{D}_{0}$, where $y_{0}^{p}=g$. If there exists an element $y_{0}^{*}$ in $\AA\left(y_{0}\right)$ such that $\left(y_{0}^{*}\right)^{p}=\bar{g}$, it was shown in JA that $\mathfrak{D}_{0}=\mathfrak{D} \times \mathfrak{R}$ implies that $\mathfrak{D}$ is cyclic over $\mathfrak{F}$. We thus assume that the ring $\Re\left[y_{0}, y_{0}^{*}\right]=\mathfrak{N}$, of dimension $2 p^{2}$ over $\mathfrak{F}$ defined by $y_{0}^{p}=g$, $\left(y_{0}^{*}\right)^{p}=\bar{g}$, is a field.

The field $\mathfrak{R}$ now has degree $p^{2}$ over $\Re$, degree $2 p^{2}$ over $\mathfrak{F}$, and $\Re$ is the maximal separable subfield over $\mathfrak{F}$ of $\mathfrak{N}$. The mapping over $\mathfrak{F}$ of $\mathfrak{\Re}$ induced by

\footnotetext{
1 The research of this paper was supported in part by a National Science Foundation grant.

2 This retiring Presidential Address was delivered at the Seventy Fourth Annual Meeting of the Society on January 23, 1967, in San Francisco.

${ }^{3}$ New results on associative division algebras, J. Algebra 5 (1967), 110-132. We shall refer to this paper as JA.
} 
(1)

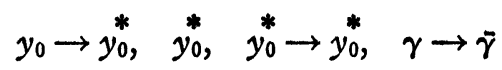

for every $\gamma$ of $\Omega$, is an extension to $\Re$ of the conjugacy automorphism of $\Re$ over $\mathfrak{F}$, and we may write every $\psi$ in $\Re$ as

$$
\psi=\sum_{i=0}^{p-1} \sum_{j=0}^{p-1} \psi_{i j} y_{0}^{i}\left(y_{0}^{*}\right)^{j} \quad\left(\psi_{i j} \text { in } \Re\right),
$$

and have

$$
\psi^{*}=\sum_{i=0}^{p-1} \sum_{j=0}^{p-1} \bar{\psi}_{i j}\left(y_{0}^{*}\right)^{i} y_{0}^{j} .
$$

Then the automorphism $\psi \rightarrow \psi^{*}$ has period two over $\mathfrak{F}$.

The field $\mathfrak{N}$ is also generated over $\mathfrak{F}$ by

$$
y=y_{0} y_{0}^{*}, \quad y^{\prime}=y_{0}\left(y_{0}^{*}\right)^{-1},
$$

where

$$
y^{p}=g \bar{g}, \quad\left(y^{\prime}\right)^{p}=g(\bar{g})^{-1} .
$$

Evidently $y^{*}=y$, that is, $y^{*}$ is a symmetric element of $\mathfrak{N}$ relative to the automorphism $\psi \rightarrow \psi^{*}$. However,

$$
\left(y^{\prime}\right)^{*}=\left(y^{\prime}\right)^{-1} \text {. }
$$

The field $\mathfrak{N}$ is clearly also generated by

$$
y_{1}=y_{0}^{2}=y y^{\prime}, \quad y_{1}^{*}=\left(y_{0}^{*}\right)^{2}=y\left(y^{\prime}\right)^{-1} .
$$

It will prove convenient later to use these generators. We note that $y_{1}^{p}=g^{2},\left(y_{1}^{*}\right)^{p}=\bar{g}^{2}$.

The ring $\mathfrak{N}$ has a pair of derivations $D$ and $D^{\prime}$ over $\Re$ induced by the mappings

$$
y D=y, \quad y^{\prime} D=y D^{\prime}=0, \quad y^{\prime} D^{\prime}=y^{\prime},
$$

and clearly $D D^{\prime}=D^{\prime} D$. If $m=\alpha y^{i} y^{\prime j}$ is any monomial in $\mathfrak{R}$ with $\alpha$ in $\Omega$, we have

$$
m D=i m, \quad m D^{\prime}=j m, \quad m^{*}=\alpha^{*} y^{i}\left(y^{\prime}\right)^{-j} .
$$

Then

(10) $\quad m^{*} D=i m^{*}=(m D)^{*}, \quad m^{*} D^{\prime}=-j m^{*}=-\left(m D^{\prime}\right)^{*}$.

Since $D$ and $D^{\prime}$ are linear transformations over $\Omega$ we conclude that

$$
(\psi D)^{*}=\psi^{*} D, \quad\left(\psi D^{\prime}\right)^{*}=-\psi^{*} D^{\prime},
$$

for every $\psi$ in $\Re$. 
Suppose that $\phi$ and $\phi^{\prime}$ are elements in $\mathfrak{N}$ so we can write

$$
\phi=\sum_{i, j=0}^{p-1} \phi_{i j} y^{i} y^{\prime j}, \quad \phi^{\prime}=\sum_{i, j=0}^{p-1} \phi_{i j}^{\prime} y^{i}\left(y^{\prime}\right)^{j},
$$

for $\phi_{i j}$ and $\phi_{i j}^{\prime}$ in $\Re$. Then it should be clear that

$$
\phi D^{p-1}=\sum_{i, j=0}^{p-1} i^{p-1} \phi_{i j} y^{i} y^{\prime j}=\sum_{i=1}^{p-1} \sum_{j=0}^{p-1} \phi_{i j} y^{i} y^{\prime j} .
$$

Similarly $\phi^{\prime} D^{\prime p-1}=\sum_{i=0}^{p-1} \sum_{j=1}^{p-1} \phi_{i,}^{\prime} y^{i} y^{\prime j}$. Thus the conditions

$$
\phi=\phi D^{p-1}, \quad \phi^{\prime}=\phi^{\prime} D^{\prime p-1}
$$

are equivalent to the conditions $\phi=\sum_{i=1}^{p-1} \sum_{j=0}^{p-1} \phi_{i j} y^{i} y^{j j}, \phi^{\prime}$ $=\sum_{i=0}^{p-1} \sum_{j=1}^{p-1} \phi_{i j}^{\prime} y^{i} y^{\prime j}$, as in JA.

We now define two additional derivations of $\mathfrak{N}$ by

$$
E=1 / 2\left(D+D^{\prime}\right), \quad E^{*}=1 / 2\left(D-D^{\prime}\right) .
$$

Then a direct computation shows that

$$
y_{1} E=y_{1}, \quad y_{1}^{*} E=y_{1} E^{*}=0, \quad y_{1}^{*} E^{*}=\left(y_{1} E\right)^{*}=y_{1}^{*} .
$$

It follows that $\Re\left(y_{1}\right)$ is the set of all elements $\psi$ in $\Re$ such that $\psi E^{*}=0$, $\Re\left(y_{1}^{*}\right)$ is the set of all elements $\psi$ in $\Re$ such that $\psi E=0$, and

$$
(\psi E)^{*}=\psi^{*} E^{*}, \quad\left(\psi E^{*}\right)^{*}=\psi^{*} E .
$$

We now turn to a reformulation of the main result of JA.

3. The principal theorem. Let us call an element $\tau$ of $\mathfrak{R}$ almost admissible if

$$
\tau E^{*}=-\tau^{*} E,
$$

that is, if $\tau E^{*}$ is a skew element of $\mathfrak{R}$. We shall also define two elements $\phi$ and $\phi^{\prime}$ in $\mathfrak{R}$ by

$$
\phi=\tau-\tau^{*}, \quad \phi^{\prime}=\tau+\tau^{*} .
$$

Thus

$$
\tau=1 / 2\left(\phi+\phi^{\prime}\right), \quad \tau^{*}=1 / 2\left(\phi^{\prime}-\phi\right),
$$

and

$$
\phi^{*}=-\phi, \quad \phi^{* *}=\phi^{\prime} .
$$

Compute $4\left(\tau E^{*}+\tau^{*} E\right)=\left(\phi+\phi^{\prime}\right)\left(D-D^{\prime}\right)+\left(\phi^{\prime}-\phi\right)\left(D+D^{\prime}\right)$ $=2\left(\phi^{\prime} D-\phi D^{\prime}\right)$. Then we have proved the following result. 
LEMMA 1. Let $\tau$ be an element of $\mathfrak{R}$, and define elements $\phi=-\phi^{*}$ and $\phi^{\prime}=\phi^{\prime *}$ by (18). Then $\phi D^{\prime}=\phi^{\prime} D$ if and only if $\tau$ is almost admissible.

We now turn to the condition (13). We observe that if $\xi$ and $\xi^{\prime}$ are indeterminates over $\Omega$ we know that $(\xi-\eta)^{p-1}(\xi-\eta)=(\xi-\eta)^{p}$ $=\xi^{p}-\eta^{p}=(\xi-\eta)\left(\xi^{p-1}+\xi^{p-2} \eta+\cdots+\xi \eta^{p-2}+\eta^{p-1}\right)$. It follows immediately that we have the identity

$$
(\xi-\eta)^{p-1}=\xi^{p-1}+\xi^{p-2} \eta+\cdots+\xi \eta^{p-2}+\eta^{p-1} .
$$

This implies that

$$
\left(E-E^{*}\right)^{p-1}=E^{p-1}+E^{p-2} E^{*}+\cdots+E E^{* p-2}+E^{* p-1}
$$

If $\tau$ is almost admissible we have

$$
\tau\left(E-E^{*}\right)^{p-1}=\tau E^{p-1}+\tau E^{*} G,
$$

where

$$
G=E^{p-2}+E^{p-3} E^{*}+\cdots+E E^{* p-8}+E^{* p-2} .
$$

Also

$$
\tau^{*}\left(E-E^{*}\right)^{p-1}=\tau^{*} E^{* p-1}+\tau^{*} E G .
$$

Hence the fact that $D^{\prime}=E-E^{*}$ implies that

$$
\phi^{\prime} D^{\prime p-1}=\tau E^{p-1}+\left(\tau E^{p-1}\right)^{*} .
$$

We know similarly that

$$
\begin{aligned}
\left(E+E^{*}\right)^{p-1}= & E^{p-1}-E^{p-2} E^{*}+\cdots \\
& +(-1)^{i} E^{p-i} E^{* i}+\cdots+E^{* p-1}
\end{aligned}
$$

Then

$$
\tau\left(E+E^{*}\right)^{p-1}=\tau E^{p-1}+\tau E^{*} G^{\prime}
$$

where

$$
G^{\prime}=-E^{p-2}+E^{p-8} E^{*}+\cdots+(-1)^{i+1} E^{p-i} E^{* i}+\cdots+E^{* p-2} .
$$

Also

$$
\tau^{*}\left(E+E^{*}\right)^{p-1}=\tau^{*} E^{* p-1}+\tau E^{*}\left(-G^{\prime}\right) .
$$

Then if $\tau$ is almost admissible, we have

$$
\phi D^{p-1}=\left(\tau-\tau^{*}\right)\left(E+E^{*}\right)^{p-1}=\tau E^{p-1}-\left(\tau E^{p-1}\right)^{*} .
$$

If $\tau=\tau E^{p-1}$ we have $\phi D^{p-1}=\tau-\tau^{*}=\phi, \phi^{\prime} D^{p-1}=\tau+\tau^{*}=\phi^{\prime}$. Con- 
versely, if $\phi D^{p-1}=\phi$ and $\phi^{\prime} D^{p-1}=\phi^{\prime}$ then $\tau-\tau^{*}=\tau E^{p-1}-\left(\tau E^{p-1}\right)^{*}$, and $\tau+\tau^{*}=\tau E^{p-1}+\left(\tau E^{p-1}\right)^{*}$, so that $\tau=\tau E^{p-1}$.

If $\tau$ is almost admissible the element $\left(\tau-\tau E^{p-1}\right) E=\tau\left(E-E^{p}\right)=0$, $\left(\tau-\tau E^{p-1}\right) E^{*}=\tau E^{*}\left(I-E^{p-1}\right)=-\tau^{*} E\left(I-E^{p-1}\right)=0$. Hence $\tau-\tau E^{p-1}$ is in $\Omega$, and the element $\tau E^{p-1}=\tau-\left(\tau-\tau E^{p-1}\right)$ differs from $\tau$ by the element $\tau-\tau E^{p-1}$ in $\Omega$. Then $\left(\tau E^{p-1}\right) E^{*}=\tau E^{*}, \quad\left(\tau E^{p-1}\right)^{*}=\tau^{*}$ $-\left(\tau-\tau E^{p-1}\right)^{*}$ and $\left(\tau E^{p-1}\right)^{*} E=\tau^{*} E=-\tau E^{*}=-\left(\tau E^{p-1}\right) E^{*}$. We state this result as follows.

Lemma 2. If $\tau$ is almost admissible, the element $\tau-\tau E^{p-1}$ is in $\Omega$, and $\tau-\left(\tau-\tau E^{p-1}\right)=\tau E^{p-1}$ is admissible.

We will now restate the principal theorem of JA. We consider a cyclic field $\mathfrak{S}$ of degree $p$ over $\Omega$ with generating automorphism $h \rightarrow h S$ over $\AA$, for every $h$ of $\mathfrak{E}$. We then define a cyclic algebra

$$
\mathfrak{D}_{0}=\left(\mathfrak{E}, y_{0}, g\right)=\mathfrak{S}+\mathfrak{S} y_{0}+\cdots+\mathfrak{S} y_{0}^{p-1},
$$

where $y_{0}^{p}=g$, and $y_{0} h=(h S) y_{0}$ for every $h$ in $\mathfrak{G}$. We assume than $\mathfrak{N}=\Re\left(y_{0}, y_{0}^{*}\right)$ is a field, and may now restate the principal theorem as follows.

THEOREM 1. The algebra $\mathfrak{D}_{0}$ is the direct product $\mathfrak{D} \times \Omega$, of $\Re$ and a central division algebra $\mathfrak{D}$ over $\mathfrak{F}$, if and only if there is an element $x$ in $\mathfrak{S}$ and an admissible element $\tau$ in $\mathfrak{R}$ such that $x^{p}-x-\tau^{p}=y_{0} x$ $-(x+1) y_{0}=0$.

We shall now reproduce the essentials of the machinery which led to the proof of this major theorem. We define two cyclic algebras

$$
\mathfrak{D}_{1}=(\mathfrak{g}, y, g \bar{g}), \quad \mathfrak{D}_{2}=\left(\mathfrak{g}^{\prime}, y^{\prime}, g / \bar{g}\right)
$$

over $\Re$, where

$$
\mathfrak{S}=\Re(x), \quad \mathfrak{S}^{\prime}=\Re\left(x^{\prime}\right), \quad x^{p}-x=\left(x^{\prime}\right)^{p}-x^{\prime}=a
$$

is in $\Re$. Thus $\mathfrak{S}$ and $\mathfrak{S}^{\prime}$ are isomorphic over $\Re$.

Define a central simple algebra $\mathfrak{B}$ over $\Re$ by

$$
\mathfrak{B}=\mathfrak{D}_{1} \times \mathfrak{D}_{2} \text {. }
$$

The algebra $\mathfrak{B}$ can then be expressed in the form

$$
\mathfrak{B}=\mathfrak{M}_{p} \times\left(\mathfrak{S}^{\prime}, y_{1}, g^{2}\right),
$$

where $y_{1}=y y^{\prime}$. Here $\mathfrak{M}_{p}$ is the total matric algebra of degree $p$ over $\mathfrak{F}$ defined as

$$
\mathfrak{M}=\mathfrak{M}_{p}=\left(\mathfrak{S}_{0}, y, g \bar{g}\right), \mathfrak{S}_{0}=\mathfrak{F}\left[x_{0}\right], x_{0}^{p}=x_{0}=\left(x-x^{\prime}\right) .
$$


By (35) we see that $x^{\prime} x_{0}-x_{0} x^{\prime}=x^{\prime} y-y x^{\prime}=0$, and so $x^{\prime}$ is in the algebra $\mathfrak{B}^{\mathfrak{R}}$ of all elements of $\mathfrak{B}$ which commute with every element of $\mathfrak{M}$. Also $y_{1} y=y y_{1}$, and $y_{1}\left(x-x^{\prime}\right)=y y^{\prime}\left(x-x^{\prime}\right)=(x+1) y y^{\prime}$ $-\left(x^{\prime}+1\right) y y^{\prime}=\left(x-x^{\prime}\right) y y^{\prime}=\left(x-x^{\prime}\right) y_{1}$, and so $y_{1}$ is in $\mathfrak{B}^{\mathfrak{M}}$. But the algebra $\left(\mathfrak{S}^{\prime}, y_{1}, g^{2}\right)$ is isomorphic to $\mathfrak{D}_{0}^{2}$ and is central simple. Hence (36) holds and may also be written in the equivalent form

$$
\mathfrak{B}=\mathfrak{M} \times \mathfrak{D}_{0}^{2} \text {. }
$$

The assumption that $\mathfrak{D}_{0}=\mathfrak{D} \times \Re$ implies that

$$
\mathfrak{B}=\mathfrak{D}_{1}^{*} \times \mathfrak{D}_{2}^{*} \text {, }
$$

where

$$
\mathfrak{D}_{1}^{*}=\left(\mathfrak{S}^{*}, y, g \bar{g}\right),
$$

for $\mathfrak{S}^{*}=\Re\left(x^{*}\right), y x=(x+1) y, x^{* p}=x^{*}+\bar{a}$ where $y$ is the element in $\mathfrak{D}_{1}$ of (33). Also

$$
\mathfrak{D}_{2}^{*}=\left(\mathfrak{S}^{\prime *}, y^{\prime-1}, \vec{g} / g\right),
$$

where $\mathfrak{S}^{\prime *}=\Re\left(x^{\prime *}\right),\left(x^{\prime *}\right)^{p}=\left(x^{*}\right)+\bar{a}, y^{\prime-1}\left(x^{\prime *}\right)=\left(x^{\prime *}+1\right) y^{\prime-1}, y^{\prime}$ is the element $y^{\prime}$ in $\mathfrak{D}_{2}$ of (33). Then it can be shown that there exist elements $\phi$ and $\phi^{\prime}$ in $\mathfrak{N}=\Re\left(y, y^{\prime}\right)$ such that

$$
x^{*}=x-\phi, \quad\left(x^{\prime}\right)^{*}=\phi^{\prime}-x^{\prime} .
$$

By the arguments already given it can be shown that $\tau=1 / 2\left(\phi+\phi^{*}\right)$ is almost admissible, and an easy normalization results in an admissible $\tau$ with the properties of Theorem 1 .

The mapping $J$ over $\mathfrak{F}$ of $\mathfrak{B}$ induced by the definitions

$$
\begin{aligned}
& x J=x^{*}, \quad\left(x^{\prime}\right) J=\left(x^{\prime}\right)^{*}, \\
& y J=y, \quad\left(y^{\prime}\right) J=\left(y^{\prime}\right)^{-1}, \quad \gamma J=\gamma^{*}=\dot{\gamma}
\end{aligned}
$$

for every element $\gamma$ in $\Re$, is an automorphism of $\mathfrak{B}$. It can then be shown that there exists a construction which imbeds $\mathfrak{B}$ in an algebra $\mathfrak{A}$ which is central simple of degree $2 p^{2}$ over $\mathfrak{F}$, where

$$
\mathfrak{A}=\mathfrak{B}+\mathfrak{B} y_{J},
$$

for an element $y_{J}$ such that, for every element $b$ in $\mathfrak{B}$, we have

$$
y_{J} b=(b J) y_{J}, \quad y_{J}^{2}=1,
$$

where then $\mathfrak{A}$ contains the total matric algebra

$$
\mathfrak{M}_{2}=\left(\Omega, y_{J}, 1\right)
$$


in which $y_{J} u=-u y_{J}$. Thus

$$
\mathfrak{A}=\mathfrak{M}_{p} \times \mathfrak{M}_{20} \times \mathfrak{D}^{2},
$$

for a total matric algebra $\mathfrak{M}_{20}$ isomorphic to $\mathfrak{M}_{2}$ and containing $\mathfrak{R}_{\text {. }}$ Indeed we can write

$$
\mathfrak{M}_{20}=(\Omega, v, 1), \quad v^{2}=1, \quad v u=-u v .
$$

However, in general, $v \neq y_{J}$. It now follows that

$$
\mathfrak{U}=\mathfrak{M}_{p 0} \times \mathfrak{A P}^{p_{0}},
$$

where $\mathfrak{M}_{p 0}$ is the total matric algebra of degree $p$ over $\mathfrak{F}$ generated by $x_{0}=x-x$ and $y$, and $\mathfrak{A}^{\mathfrak{M}_{p_{0}}}$ is the set of all elements of $\mathfrak{A}$ which commute with $x_{0}$ and $y$. Then

$$
\mathfrak{U}^{\mathfrak{M}_{p 0}}=\mathfrak{D}^{2} \times \mathfrak{M}_{20}=\mathfrak{D}_{0}^{2}+\mathfrak{D}_{0}^{2} v \text {. }
$$

We shall now derive a property which is actually the result of an effort to find the element $v$ in the corresponding algebra $\mathfrak{M}_{20}$, and hence the algebra $\mathfrak{D}^{2}$ over $\mathfrak{F}$ of $(50)$.

3. Determination of $q(\delta)$. We first consider the set $\mathbb{C}$ of all elements of $\mathfrak{A}$ which commute with $y$. This is clearly the vector space

$$
\mathfrak{C}=\left[\mathfrak{D}_{0}^{2} \times \Re(y)\right]+\left[\mathfrak{D}_{0}^{2} \times \Re(y)\right] y_{J},
$$

where it will be convenient to use the notations

$$
\begin{gathered}
z=x^{\prime}, \quad \mathfrak{S}^{\prime}=\Re(z), \quad z^{p}-z=a, \\
\mathfrak{D}_{0}^{2}=\left(\mathfrak{S}^{\prime}, y_{1}, g^{2}\right), \quad y_{1} z=(z+1) y_{1} .
\end{gathered}
$$

The elements of $\mathfrak{D}_{0}^{2} \times \mathfrak{R}(y)$ which commute with $x_{0}=x-x^{\prime}$ are those which are in $\mathfrak{D}_{0}^{2}$, and all of these elements commute with $u$. Then the elements of $\mathbb{E}$ which anticommute with $u$ are exclusively those in $\left[\mathfrak{D}_{0}^{2} \times \Re(y)\right] y_{J}$. Every such element has the form

$$
q=\left(\delta_{0}+z \delta_{1}+z^{2} \delta_{2}+\cdots+z^{p-1} \delta_{p-1}\right) y_{J},
$$

for $\delta_{0}, \cdots, \delta_{p-1}$ in $\Re=\Re\left[y, y^{\prime}\right]=\Re\left(y, y_{1}\right)=\Re\left(y_{1}, y_{1}^{*}\right)$. Note that

$$
\delta z=z \delta+\delta D^{\prime}, \quad \psi z=z \psi+\psi E
$$

for every $\delta$ of $\Re$ and $\psi$ in $\Re\left(y_{1}\right)$, since $y_{1} E=y_{1} D=y_{1}$. We are, of course only interested in those elements $q$ of (53) which commute with $x_{0}$. The dimension of $\mathfrak{D}_{0}^{2}$ v over $\mathfrak{F}$ is $2 p^{2}$ since $\mathfrak{D}_{0}^{2}$ has dimension $p^{2}$ over $\Re$. This is the same as the dimension of $\mathfrak{N}$ over $\mathfrak{F}$. We shall actually show that there is a linear mapping 


$$
\delta \rightarrow q(\delta)
$$

over $\mathfrak{F}$ of $\delta$ into the set $\mathfrak{D}_{0}^{2} v$ and also that $q(\delta)=0$ if and only if $\delta=0$ so that it will follow that this mapping is one-to-one onto. We state this property as follows.

THEOREM 2. An element $q$ in $\left[\mathfrak{D}_{0}^{2} \times \mathfrak{\Omega}(y)\right] y_{J}$ commutes with $x_{0}$, and so is in $\mathfrak{D}_{0}^{2} v$, if and only if $q=q(\delta)$ for $\delta$ in $\mathfrak{N}$, where $q(\delta)$ is defined by (53) for

$$
\begin{gathered}
\delta=\delta_{p-1}, \delta_{p-2}=\delta \Delta, \cdots, \delta_{p-i}=\delta \Delta^{i-1}, \cdots, \delta_{1}=\delta \Delta^{p-2}, \\
\delta_{0}=\delta\left(\Delta^{p-1}-I\right) .
\end{gathered}
$$

Here $\Delta$ is a linear transformation over $\Re$ of $\mathfrak{N}$ defined by

$$
\delta \Delta=\delta \tau-\delta E,
$$

where $\tau$ is the admissible element of Theorem 1. The mapping $\delta \rightarrow q(\delta)$ is one-to-one onto and

$$
\Delta^{p}-\Delta=\tau^{p} I
$$

For the condition $q(x-z)=(x-z) q$ is equivalent to $(x-z) q y_{J}$ $=q y_{J}\left(x^{*}-z^{*}\right)=q y_{J}\left(x+z-\phi-\phi^{\prime}\right)$. Thus

$$
\left(q y_{J}\right) x-x\left(q y_{J}\right)+\left(q y_{J}\right) z+z\left(q y_{J}\right)=q y_{J}\left(\phi+\phi^{\prime}\right),
$$

where $q y_{J}=\delta_{0}+z \delta_{1}+\cdots+z^{p-1} \delta_{p-1}$ is in $\mathfrak{D}_{0}^{2} \times \Re(y)$. But

$$
\left(q y_{J}\right) x-x\left(q y_{J}\right)=\sum_{i=0}^{p-1} z^{i}\left(\delta_{i} x-x \delta_{i}\right)=\sum_{i=0}^{p-1} z^{i}\left(\delta_{i} D\right),
$$

and

$$
\left(q y_{J}\right) z+z\left(q y_{J}\right)=\sum_{i=0}^{p-1} z^{i}\left(\delta_{i} z+z \delta_{i}\right)=\sum_{i=0}^{p-1}\left[2 z^{i+1} \delta_{i}+z^{i}\left(\delta_{i} D^{\prime}\right)\right] .
$$

It follows that $q$ commutes with $x_{0}$ if and only if

$$
\sum_{i=0}^{p-1} z^{i+1} \delta_{i}=\sum_{i=0}^{p-1} z^{i}\left(\delta_{i} \Delta\right)
$$

We put $\delta=\delta_{p-1}$ and obtain

$$
\delta_{i-1}=\delta_{i} \Delta=\delta \Delta^{p-i} \quad(i=2, \cdots, p-1)
$$

from (60), as well as

$$
\delta_{0}+\delta=\delta_{1} \Delta, \quad \delta \tau^{p}=\delta\left(z^{p}-z\right)=\delta_{0} \Delta .
$$


Since (61) yields $\delta_{1} \Delta=\delta \Delta^{p-1}$ we have $\delta_{0}=\delta\left(\Delta^{p-1}-I\right), \delta\left(\Delta^{p}-\Delta-\tau^{p}\right)=0$. The condition (56) certainly implies that the mapping $\delta \rightarrow q(\delta)$ of $\mathfrak{N}$ on to $\mathfrak{D}_{0}^{2} v$ is linear over $\mathfrak{F}$. By our dimension argument this mapping is one-to-one onto, and so $\delta\left(\Delta^{p}-\Delta-\tau^{p} I\right)=0$ for every $\delta$, and (58) holds.

4. A representation theorem. We have seen that

$$
\mathfrak{D}_{0}^{2} v=\left(\mathfrak{D}^{2}+\mathfrak{D}^{2} u\right) v=v\left(\mathfrak{D}^{2}-\mathfrak{D}^{2} u\right)=\mathfrak{D}_{0}^{2} \text {. }
$$

Since $\mathfrak{D}_{0}^{2}$ is a division algebra we see that

$$
\mathfrak{D}_{0}^{2}\left(\mathfrak{D}_{0}^{2} v\right)=\left(\mathfrak{D}_{0}^{2} v\right) \mathfrak{D}_{0}^{2}=\mathfrak{D}_{0}^{2} v \text {. }
$$

Thus, for every $\delta$ in $\mathfrak{N}$ and $f$ in $\mathfrak{D}_{0}^{2}$, there exist $\delta^{\prime}$ and $\delta^{\prime \prime}$ in $\mathfrak{N}$ such that

$$
f q(\delta)=q\left(\delta^{\prime}\right), \quad q(\delta) f=q\left(\delta^{\prime \prime}\right) .
$$

Let us now compute $\delta^{\prime}$ and $\delta^{\prime \prime}$ for the generators $z$ and $y_{1}$ of $\mathfrak{D}_{0}^{2}$.

We see first that $z q(\delta) y_{J}=z\left(\delta_{0}+z \delta_{1}+\cdots+z^{p-1} \delta\right)=z \delta_{0}+z^{2} \delta_{1}$ $+\cdots+z^{p-1} \delta_{p-2}+\left(z+\tau^{p}\right) \delta$. Thus the coefficient of $z^{p-1}$ in $q\left(\delta^{\prime}\right)$ is $\delta_{p-2}=\delta \Delta$ and we have $z q(\delta)=q(\delta \Delta)$. It follows that

$$
z^{i} q(\delta)=q\left(\delta \Delta^{i}\right) \quad(i=0, \cdots, p-1) .
$$

We also see that

$$
\begin{aligned}
q(\delta) z & =\left[q(\delta) y_{J}\right] z^{*} y_{J}=\left[\left(\delta_{0}+z \delta_{1}+\cdots+z^{p-1} \delta_{p-1}\right)\left(\phi^{\prime}-z\right)\right] y_{J} \\
& =\left(\sum_{i=0}^{p-1} z^{i} \delta_{i} \phi^{\prime}-z^{i} \delta_{i} z\right) y_{J}=\sum_{i=0}^{p-1} z^{i} \delta_{i} \phi^{\prime}-z^{i+1} \delta_{i}-z^{i} \delta_{i} D^{\prime}
\end{aligned}
$$

The coefficient on the right of $z^{p-1}$ is the $\delta^{\prime \prime}$ for $z$ and this is clearly

$$
\delta \phi^{\prime}-\delta \Delta-\delta D^{\prime}=\delta \Delta^{\prime}=\delta R\left(\phi^{\prime}\right)-\delta \cdot 1 / 2\left[R\left(\phi+\phi^{\prime}\right)-\left(D+D^{\prime}\right)\right]-\delta D^{\prime} .
$$

Hence

$$
q(\delta) z^{i}=q\left(\delta \Delta^{\prime i}\right)
$$

where

$$
\delta \Delta^{\prime}=\delta \tau^{*}+\delta E^{*} .
$$

Since $[z q(\delta)] z=z[q(\delta) z]$ we have derived the commutativity relation

$$
\Delta \Delta^{\prime}=\Delta^{\prime} \Delta \text {. }
$$

We next observe that $y_{1} z^{i}=(z+1)^{i} y_{1}$, and so the only term involving $z^{p-1}$ in $y_{1} \sum_{i=0}^{p-1} z^{i} \delta_{i}=\sum_{i=0}^{p-1}(z+1)^{i} \delta_{i} y_{1}$ is $z^{p-1} \delta y_{1}$. Hence 


$$
\psi\left(y_{1}\right) q(\delta)=q(\delta \psi)
$$

for every $\psi=\psi\left(y_{1}\right)$ in $\AA\left(y_{1}\right)$. We also note that $q(\delta) \cdot \psi=\sum_{i=0}^{p-1} z^{i} \delta_{i}\left(y_{J} \psi\right)$ $=\sum_{i-0}^{p-1} z^{i}\left(\delta_{i} \psi^{*}\right) y_{J}$, and thus that

$$
q(\delta) \psi=q\left(\delta \psi^{*}\right)
$$

for every $\psi$ in $\Re\left(y_{1}\right)$. Thus $z\left[q(\delta) y_{1}\right]=z q\left(\delta y_{1}^{*}\right)=q\left[\left(\delta y_{1}^{*}\right) \Delta\right]=[z q(\delta)] y_{1}$ $=q\left(\delta \Delta y_{1}^{*}\right)$, and it follows that $\Delta R\left(y_{1}^{*}\right)=R\left(y_{1}^{*}\right) \Delta$. Similarly $\Delta^{\prime} R\left(y_{1}\right)$ $=R\left(y_{1}\right) \Delta^{\prime}$ and so

$$
\Delta R_{\theta}-R_{\theta} \Delta=\Delta^{\prime} R_{\psi}-R_{\psi} \Delta^{\prime}=0,
$$

for every $\psi$ in $\Omega\left(y_{1}\right)$ and $\theta$ in $\Omega\left(y_{1}^{*}\right)$. We are also aware of the fact that $E$ and $E^{*}$ are derivations of $\mathfrak{N}$ such that $\psi E^{*}=\psi^{*} E=0$ for every $\psi$ in $\Omega\left(y_{1}\right)$. But $(\delta \psi) \Delta=(\delta \psi)\left(R_{\tau}-E\right)=\delta \tau \psi-(\delta \psi) E=\delta \tau \psi$ $-[\delta E \psi+\delta(\psi E)]$. Hence

$$
R_{\psi} \Delta=\Delta R_{\psi}-R_{\psi E} .
$$

Similarly $(\delta \psi) \Delta=\delta \psi \tau^{*}+(\delta \psi) E^{*}=\left(\delta \tau^{*}\right) \psi+\left(\delta E^{*}\right) \psi+\delta \psi E^{*}$, and so

$$
R_{\psi} \Delta^{\prime}=\Delta^{\prime} R_{\psi}+R_{\psi W}^{*} \text {. }
$$

It follows that

$$
R\left(y_{1}\right)(\Delta+I)=\Delta R\left(y_{1}\right), \quad R\left(y_{1}\right) \Delta^{\prime}=\Delta^{\prime} R\left(y_{1}\right),
$$

and that

$$
R\left(y_{1}^{*}\right) \Delta=\Delta R\left(y_{1}^{*}\right), \quad R\left(y_{1}^{*}\right) \Delta^{\prime}=\left(\Delta^{\prime}+I\right) R\left(y_{1}^{*}\right) .
$$

It now follows that the algebra $\mathfrak{D}_{3}$ of linear transformations over $\Re$ on $\mathfrak{R}$ generated by $\Delta$ and the right multiplication $R\left(y_{1}\right)$, is antiisomorphic to $\mathfrak{D}_{0}^{2}$. Since $q(\delta)\left(z^{p}-z\right)=q\left[\delta\left(\Delta^{\prime} p-\Delta^{\prime}\right)\right]=q\left(\delta \tau^{* p}\right)$ we have

$$
\Delta^{\prime p}-\Delta^{\prime}=\left(\tau^{*}\right)^{p} I
$$

Hence the algebra $\mathfrak{D}_{4}$ of linear transformations on $\mathfrak{N}$ generated by $\Delta^{\prime}$ and $R\left(y_{1}^{*}\right)$ is isomorphic to $\left(\mathfrak{D}_{0}^{2}\right)^{*}=\left(\mathfrak{S}^{*}, y_{1}^{*}, \mathfrak{z}^{2}\right)$, where $\mathfrak{S}^{*}=\mathfrak{A}^{*}\left(z^{*}\right)$, and $y_{1}^{*} z^{*}=\left(z^{*}+1\right) y_{1}^{*}, y_{1}^{* p}=\bar{g}^{2}$. Since $\mathfrak{N}$ has dimension $p^{2}$ over $\Re$, and the algebra of all linear transformations over $\Re$ of $\Re$ has degree $p^{2}$ over $\mathfrak{N}$, it must be equal to the direct product $\mathfrak{D}_{3} \times \mathfrak{D}_{4}$. These algebras, $\mathfrak{D}_{3}$ and $\mathfrak{D}_{4}$, are in the direct product relation by (69), (75), (76) and the fact that right multiplications commute. We have derived the following representation theorem.

THEOREM 3. The algebra $\mathfrak{D}_{3}$ generated by $\Delta$ and $R\left(y_{1}\right)$ is a representation by linear transformations on $\mathfrak{N}$ of $\left(\mathfrak{D}_{0}^{2}\right)^{-1}$. The algebra $\mathfrak{D}_{4}$, generated by $\Delta^{\prime}$ and $R\left(y_{1}^{*}\right)$, is a representation by linear transformations on 
$\mathfrak{N}$ of $\left(\mathfrak{D}_{0}^{2}\right)^{*}$. These representations are in the direct product relation; $\mathfrak{D}_{3} \times \mathfrak{D}_{4}$ is the full matric algebra of all linear transformations on $\mathfrak{N}$.

This result is another verification of the fact already derived that $\mathfrak{D}_{\theta}$ and $\mathfrak{D}_{0}^{*}$ are isomorphic under the assumption $x^{p}-x=\tau^{p}$ for $\tau$ admissible.

5. A cyclicity condition. We have now seen that, if $d_{0}, \cdots$, $d_{p-1}$ are in $\Omega\left(y_{1}\right)$ and

$$
d=d_{0}+z d_{1}+\cdots+z^{p-1} d_{p-1}
$$

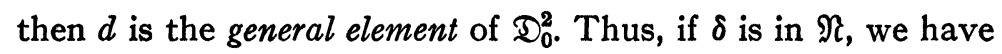

$$
d q(\delta)=q\left(\delta^{\prime}\right), \quad q(\delta) d=q\left(\delta^{\prime \prime}\right)
$$

where

$$
\delta^{\prime}=\delta d_{0}+\left(\delta d_{1}\right) \Delta+\cdots+\left(\delta d_{p-1}\right) \Delta^{p-1}
$$

and

$$
\delta^{\prime \prime}=\delta d_{0}^{*}+\left(\delta \Delta^{\prime}\right) d_{1}^{*}+\cdots+\left(\delta \Delta^{\prime p-1}\right) d_{p-1}^{*} .
$$

Moreover, there is an element $\epsilon$ in $\mathfrak{N}$ such that

$$
v=q(\epsilon), \quad v^{2}=1,
$$

and $\mathfrak{D}^{2}$ is the set of all elements $d$ of (78) such that $d v=v d$.

As a consequence of the results above we see that the algebra $\mathfrak{D}$ is cyclic if and only if there exists an element $d$ of (78) such that

$$
d v=v d, \quad d^{p}=\gamma \quad(\gamma \text { in } \Omega) .
$$

The element $\epsilon$ is far from easy to compute, if $\tau$ is given, even when $p=3$. However, we can derive the following result.

TheOREM 4. The algebra $\mathfrak{D}$ defined by an admissible $\tau$ is cyclic over $\mathfrak{F}$ if and only if there exists an element $d=d_{0}+z d_{1}+\cdots+z^{p-1} d_{p-1}$

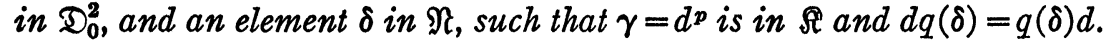

Let us observe that every $q(\delta)=f v$ for $f$ in $\mathfrak{D}_{0}^{2}$ and every $f v=q(\delta)$ for some $\delta$. Then $d q(\delta)=q(\delta) d$ if and only if $d f v=f v d,\left(f^{-1} d f\right) v=v d v$. Then $(v d v)^{p}=v d^{p} v=v \gamma v=\bar{\gamma}=f^{-1} d^{p} f=f^{-1} \gamma f=\gamma$. Hence the existence of such a $d$ and $f$ implies that there exists an element $\gamma$ in $\mathfrak{F}$ such that $\Re\left(\gamma^{1 / p}\right)$ splits $\mathfrak{D}_{0}, \mathfrak{F}\left(\gamma^{1 / p}\right)$ will split $\mathfrak{D}, \mathfrak{D}$ is cyclic. Conversely, if $\mathfrak{D}$ is cyclic over $\mathfrak{F}$ there exists an element $d$ in $\mathfrak{D}^{2}$ such that $d^{p}=\gamma$ in $\mathfrak{F}$, $d v=v d, v=q(\epsilon)$ and our theorem is proved.

If $d^{\prime}=f^{-1} d f$ for $d$ and $f$ in $\mathfrak{D}_{0}^{2}$ and $d^{\prime} q(\delta)=q(\delta) d^{\prime}$ then $f^{-1} d f q(\delta)$ $=q(\delta) f^{-1} d f,\left[f q(\delta) f^{-1}\right] d=d\left[f q(\delta) f^{-1}\right]$. Since (65) implies that $f q(\delta) f^{-1}$ 
$=q\left(\delta^{\prime}\right)$ for $\delta^{\prime}$ in $\mathfrak{N}$, we see that the replacement of $d$ by any conjugate element $f^{-1} d f$ merely alters $\delta$ and so all conjugate elements have the same role in the application of Theorem 4.

We now derive a sufficient condition for cyclicity.

TheOREM 5. Let $d=d_{0}+z d_{1}$ for $d_{0}$ and $d_{1}$ in $\Re\left(y_{1}\right)$. Then $d^{p}=\gamma$ in $\Re$ if and only if $d_{1}^{p-1} E^{p-1}=d_{1}^{p-1}$.

For the algebra $\mathfrak{D}_{0}^{2}=\left(\mathfrak{S}^{\prime}, y_{1}, g^{2}\right)$ has a representation as a set of $p$-rowed square matrices with elements in $\mathfrak{S}^{\prime}=\mathfrak{\Omega}(z)$. The diagonal elements of the representation of an element of $\mathfrak{D}_{0}^{2}$ are

$$
f_{0}(z), f_{0}(z+1), \cdots, f_{0}(z+p-1),
$$

where we are writing

$$
d=f_{0}(z)+f_{1}(z) y_{1}+\cdots+f_{p-1}(z) y_{1}^{p-1},
$$

and the $f_{i}(z)$ are in $\mathfrak{S}^{\prime}$. The trace of each such matrix representation $A=A(d)$ is the element in $\Re$ given by

$$
\operatorname{tr}(A)=f_{0}(z)+\cdots+f_{0}(z+p-1) .
$$

But, if $f_{0}(z)=f_{00}+f_{01} z+\cdots+f_{0 p-1} z^{p-1}$ for $f_{0 j}$ in $\Omega$, it is well known that

$$
\operatorname{tr}(A)=-f_{0 p-1} .
$$

It follows that, if $d$ is given by (78), the polynomial $f_{0}(z)=d$ $-\left[d_{0} E^{p-1}+z\left(d_{1} E^{p-1}\right)+\cdots+z^{p-1}\left(d_{p-1} E^{p-1}\right)\right]$ and so we have

$$
\operatorname{tr}(A)=-\left(d_{p-1}-d_{p-1} E^{p-1}\right) .
$$

It now follows that, if $d=d_{0}+z d_{1}$, then we automatically have

$$
\operatorname{tr}\left[A\left(d^{i}\right)\right]=0 \quad(i=1, \cdots, p-2) .
$$

We also see that, if $d=d_{0}+z d_{1}$, the relation $\psi z=z \psi+\psi E$ for every $\psi$ in $\Re\left(y_{1}\right)$ implies that

$$
d^{p-1}=d_{0}^{\prime}+z d_{1}^{\prime}+\cdots+z^{p-2} d_{p-2}^{\prime}+z^{p-1} d_{1}^{p-1} .
$$

Hence $\operatorname{tr}\left[A\left(d^{p-1}\right)\right]=\operatorname{tr}\left[A\left(z^{p-1} d_{1}^{p-1}\right)\right]=\operatorname{tr}\left[z\left(z^{p-1} d_{1}^{p-1} E^{p-1}-d_{1}^{p-1}\right)\right]=d_{1}^{p-1}$ $\left(E^{p-1}-I\right)$. We have shown that the relation $\operatorname{tr}\left[A\left(d^{i}\right)\right]=0$, for $i=$ $1, \cdots, p-1$, holds if and only if $d_{1}^{p-1}\left(E_{1}^{p-1}-I\right)=0$.

The Newton identities

$$
s_{k}+c_{1} s_{k-1}+\cdots+c_{k-1} s_{k}+k c_{k}=0 \quad(k=1, \cdots p-1)
$$

hold for the polynomial 


$$
\begin{aligned}
f(\xi) & =\left(\xi-\xi_{1}\right)\left(\xi-\xi_{2}\right) \cdots\left(\xi-\xi_{p}\right) \\
& =\xi^{p}+c_{1} \xi^{p-1}+\cdots+c_{p-1} \xi+c_{p},
\end{aligned}
$$

in independent indeterminates $\xi, \xi_{1}, \cdots, \xi_{p}$ over the ring of all ordinary integers. They then hold also for any field of characteristic $p$. We also see that, if

$$
s_{k}=\xi_{1}^{k}+\xi_{2}^{k}+\cdots+\xi_{p}^{k}
$$

then $s_{k}$ is indeed the trace of the matrix $A\left(d^{k}\right)$, where $A(d)$ is the matrix representation of $d$ as a matrix with elements in the field $\mathfrak{S}^{\prime}$, $f(\xi)$ is the characteristic function over $\Omega$ of $A(d)$ and of $d$. Thus, the assumption that $d=d_{0}+z d_{1}$, for $d_{0}$ and $d_{1}$ in $\Omega\left(y_{1}\right)$, implies that $f(\xi)$ $=\xi^{p}+c_{p-1} \xi+c_{k}, \quad s_{p-1}+(p-1) c_{p-1}=0, \quad c_{p-1}=0$ if and only if $d_{1}^{p-1}\left(E^{p-1}-I\right)=0$. Hence $d^{p}=\gamma$ in $\Omega$ if and only if $d_{1}^{p-1}\left(E^{p-1}-I\right)=0$.

We may now derive the following sufficient condition.

THEOREM 6. Let $\mathfrak{D}$ be a division algebra over $\mathfrak{F}$ defined by a field $\mathfrak{N}=\Re\left(y_{0}, y_{1}^{*}\right)$ and an admissible $\tau$ in $\mathfrak{R}$. Suppose that there exists $a \delta$ in $\mathfrak{N}$ and elements $d_{0}$ and $d_{1}$ in $\Re\left(y_{1}\right)$ such that $d_{1}^{p-1}\left(I-E^{p-1}\right)=0$ and

$$
d_{0}-d_{1} E+\tau^{\prime} d_{1}-\delta^{-1}(\delta E) d_{1}=d_{0}^{*}+\left(\tau^{\prime} d_{1}\right)^{*}+\delta^{-1}\left(\delta E^{*}\right) d_{1}^{*}
$$

for $\tau-\tau^{\prime}$ in $\Omega$. Then $\mathfrak{D}$ is cyclic over $\mathfrak{F}$.

For, it should be clear that if (94) holds for $\tau^{\prime}=\tau+\lambda, \lambda$ in $\Omega$, it will also hold for $\tau$, where we merely adjust the value of $d_{0}$. Also, (94) is equivalent to

$$
d q(\delta)=q(\delta) d,
$$

and this condition implies that $d^{p} q(\delta)=q(\delta) d^{p}$. We have already seen that $d_{1}^{p-1}\left(I-E^{p-1}\right)=0$ if and only if $d=d_{0}+z d_{1}$ has the property that $d^{p}=\gamma$ in $\Omega$, and Theorem 4 then implies that $\mathfrak{D}$ is cyclic. This completes our proof.

6. The case of a split $\tau$. Assume that $\tau$ is admissible and that $\tau$ is split, that is,

$$
\tau=\tau_{0} \tau_{1}^{*},
$$

for $\tau_{0}$ and $\tau_{1}$ in $\Omega\left(y_{1}\right)$. If $\tau_{1} E=0$ then $\tau_{1}$ is in $\Omega$, and so $\tau$ is in $\Re\left(y_{1}\right)$. But $\tau=\tau E^{p-1}$ and it was shown in JA that then $\mathfrak{D}$ cannot be $a$ division algebra.

Hence, let $\tau_{1} E \neq 0$. We form $\tau E^{*}=\tau_{0}\left(\tau_{1} E\right)^{*}=-\tau^{*} E=-\left(\tau_{0}^{*} \tau_{1}\right) E$ $=-\tau_{0}^{*}\left(\tau_{1} E\right)$. Then $\tau_{0}\left(\tau_{1} E\right)^{-1}=-\tau_{0}^{*}\left[\left(\tau_{1} E\right)^{*}\right]^{-1}$ is in both $\Omega\left(y_{1}\right)$ and $\Omega\left(y_{1}^{*}\right)$ and so is in $\Omega$. Thus, we may write $\tau_{0}=\lambda\left(\tau_{1} E\right)$ for $\lambda$ in $\Omega$, $\lambda=-\lambda^{*}$, and we have shown that 


$$
\tau=\alpha u\left(\tau_{1} E\right) \tau_{1}^{*} \quad(\alpha \text { in } \mathfrak{F}) .
$$

We may now write

$$
\tau_{1}=\tau_{10}+\tau_{11} y_{1}+\cdots+\tau_{1, p-1} y_{1}^{p-1} \quad\left(\tau_{1 j} \text { in } \Omega\right)
$$

so that

(99) $\tau_{1} E=y_{1} \tau_{2}=y_{1}\left[\tau_{11}+2 \tau_{12} y_{1}+\cdots+(p-1) \tau_{1, p-1} y_{1}^{p-1}\right]$.

We now put

$$
\delta=\tau_{2}^{*} y_{1}, \quad \delta E=\delta, \quad \delta^{*}=\tau_{2} y_{1}^{*}
$$

and obtain

$$
\delta \delta^{*}=\tau_{2} \tau_{2}^{*} y_{1} y_{1}^{*}=\left(\tau_{1} E\right)\left(\tau_{1} E\right)^{*}
$$

Put

$$
d_{1}=\left(\tau_{1} E\right)^{p-1}
$$

so that the product

$$
\left(\delta \delta^{*}\right) d_{1}=\left(\tau_{1} E\right)^{p}\left(\tau_{1} E\right)^{*}
$$

is in $\Omega\left(y_{1}^{*}\right)$. Then

$$
\left[\left(\delta \delta^{*}\right) d_{1}\right] E=\left[\left(\delta \delta^{*}\right) E\right] d_{1}+\left(\delta \delta^{*}\right)\left(d_{1} E\right)=0 .
$$

We have shown that

$$
\omega=\frac{\delta E}{\delta}+\frac{\delta^{*} E}{\delta^{*}}=\frac{\left(\delta \delta^{*}\right) E}{\delta \delta^{*}}=1+\frac{\tau_{2} E}{\tau_{2}}
$$

is in $\Omega\left(y_{1}\right)$ and

$$
\omega d_{1}=-d_{1} E
$$

We see that

$$
\frac{\delta^{*} E}{\delta^{*}}=\omega-1, \quad \frac{\delta E^{*}}{\delta}=\omega^{*}-1,
$$

and (94) becomes

$$
\begin{aligned}
d_{0}-d_{0}^{*}+\tau d_{1}-\left(\tau d_{1}\right)^{*} & =-\omega d_{1}+\left(\omega^{*}-1\right) d_{1}^{*}+d_{1} \\
& =\omega^{*} d_{1}^{*}-\omega d_{1}+d_{1}-d_{1}^{*} .
\end{aligned}
$$

By our construction, the elements

$$
\left(\tau d_{1}\right)^{*}=\left[\alpha u \tau_{1}^{*}\left(\tau_{1} E\right)^{p}\right]^{*}, \quad \omega d_{1}=-d_{1} E, d_{1}
$$


are all in $\Omega\left(y_{1}\right)$. Hence the value

$$
d_{0}=\left(\tau d_{1}\right)^{*}-\omega d_{1}+d_{1},
$$

yields a solution of (94). Also $d_{1}^{p-1}=\left(\tau_{1} E\right)^{t}$ where $t=p^{2}-2 p+1$ and so $d_{1}^{p-1}=\beta\left(\tau_{1} E\right)$ for $\beta$ in $\Omega, d_{1}^{p-1} E^{p-1}=\beta\left(\tau_{1} E^{p}\right)=\beta\left(\tau_{1} E\right)=d_{1}^{p-1}$, and we have derived the following result. ${ }^{4}$

THEOREM 7. If $\tau$ is the product $\tau=\tau_{0} \tau_{1}^{*}$ for polynomials $\tau_{0}$ and $\tau_{1}$ in $\Re\left(y_{1}\right)$ the algebra $\mathfrak{D}$ defined by $\mathfrak{N}$ and $\tau$ is cyclic over $\mathfrak{F}$.

7. A generalization. It should now be clear that we are using our cyclicity condition to determine values of $\tau$ for which this particular condition holds. We shall now pass to a generalization which uses this condition more deeply.

We observe that (94) may be written in the form

$$
\left(d_{0}+\tau^{\prime} d_{1}\right)-\left(d_{0}+\tau^{\prime} d_{1}\right)^{*}=d_{1} E+\delta^{-1}\left[(\delta E) d_{1}+\left(\delta E^{*}\right) d_{1}^{*}\right]
$$

This condition can hold only if $d_{1} E+\delta^{-1}\left[(\delta E) d_{1}+\left(\delta E^{*}\right) d_{1}^{*}\right]$ is skew, that is

$$
d_{1} E+\left(d_{1} E\right)^{*}+d_{1}\left(\frac{\delta E}{\delta}+\frac{\delta^{*} E}{\delta^{*}}\right)+\left(\frac{\delta E}{\delta}+\frac{\delta^{*} E}{\delta^{*}}\right)^{*} d_{1}^{*}=0
$$

We put

$$
\delta=\nu\left[\left(\nu d_{1}\right)^{*}\right]^{-1}, \quad \delta \delta^{*}=\left(d_{1} \dot{d}_{1}^{*}\right)^{-1},
$$

where $\nu$ is in $\mathfrak{N}$ and $d$ in $\Re\left(y_{1}\right)$ will be determined later. Then, if

$$
\omega=\frac{\delta E}{\delta}+\frac{\delta^{*} E}{\delta^{*}}=\frac{\left(\delta \delta^{*}\right) E}{\delta \delta^{*}},
$$

we have

$\left(\delta \delta^{*}\right) d_{1}^{*}=\left(d_{1}^{*}\right)^{-1}, \quad\left[\left(\delta \delta^{*}\right) d_{1}\right] E=\left[\left(d_{1}^{*}\right)^{-1}\right] E=\left(\delta \delta^{*}\right) E d_{1}+\left(\delta \delta^{*}\right)\left(d_{1} E\right)=0$ since $d_{1}^{*}$ is in $\Omega\left(y_{1}^{*}\right)$. It follows that $\delta \delta^{*}\left(\omega d_{1}+d_{1} E\right)=0$, that

$$
\omega d_{1}=-d_{1} E
$$

and so $\omega$ is in $\Re\left(y_{1}\right)$.

We now observe that, if $\psi$ is any element of $\mathfrak{N}$, we have

4 This theorem was first derived by the author in the case of a special value of $\tau$. A proof of the general case was then found by Charles Lanski (NSF Fellow at Chicago), using the machinery developed here. The author has not seen his proof, but it is presumably similar to that given in this section. 


$$
\begin{aligned}
\frac{\psi E}{\psi}-\frac{\psi^{*} E}{\psi^{*}} & =\frac{\psi^{*}(\psi E)-\psi\left(\psi^{*} E\right)}{\left(\psi^{*}\right)^{2}} \cdot \frac{\psi^{*}}{\psi} \\
& =\frac{\Lambda E}{\Lambda},
\end{aligned}
$$

where

$$
\Lambda=\frac{\psi}{\psi^{*}} .
$$

We now take $\nu=\psi_{0}^{\pi}$, where $p=2 \pi-1$, then

$$
\frac{\delta}{\delta^{*}}=\frac{\nu^{2} d_{1}}{\left(\nu^{2} d_{1}\right)^{*}}=\frac{\psi_{0} d_{1} \psi_{0}^{p}}{\left(\psi_{0} d_{1} \psi_{0}^{p}\right)^{*}}=\frac{\psi \lambda}{\psi^{*} \lambda^{*}},
$$

where $\lambda$ is a function of $\psi$ in $\Omega$, and the element $\psi$ is arbitrary, and thus

$$
\frac{(\psi \lambda) E}{\psi \lambda}-\frac{\left(\psi^{*} \lambda^{*}\right) E}{\psi^{*} \lambda^{*}}=\frac{\psi E}{\psi}-\frac{\psi^{*} E}{\psi^{*}}=\frac{\Lambda E}{\Lambda}=\frac{\Lambda^{\prime} E}{\Lambda^{\prime}},
$$

where $\Lambda=\psi\left(\psi^{*}\right)^{-1}$ and $\Lambda^{\prime}=\delta\left(\delta^{*}\right)^{-1}$. Hence $\delta$ can be selected so that

$$
\frac{\delta E}{\delta}-\frac{\delta^{*} E}{\delta^{*}}=\frac{\psi E}{\psi}-\frac{\psi^{*} E}{\psi^{*}},
$$

for arbitrary $\psi$ in $\mathfrak{R}$, where $\delta$ satisfies (114).

We now put

$$
\tau^{\prime}=\frac{1}{2}\left(\frac{\delta E}{\delta}-\frac{\delta^{*} E}{\delta^{*}}\right)+\tau^{\prime \prime}
$$

where

$$
\tau^{\prime \prime}=\alpha u\left(\tau_{1} E\right) \tau_{1}^{*}
$$

for $\alpha$ in $\mathfrak{F}$ and $\tau_{1}$ in $\Re\left(y_{1}\right)$. We also take

$$
d_{1}=\left(\tau_{1} E\right)^{p-1} \text {. }
$$

We have already seen that $\tau^{\prime \prime}$ is almost admissible. But

$$
\left(\frac{\delta E}{\delta}-\frac{\delta^{*} E}{\delta^{*}}\right) E^{*}=\frac{\delta\left(\delta E E^{*}\right)-\delta E\left(\delta E^{*}\right)}{\delta^{2}}-\frac{\delta^{*}\left(\delta^{*} E E^{*}\right)-\delta^{*} E\left(\delta^{*} E^{*}\right)}{\left(\delta^{*}\right)^{2}}
$$

is clearly skew. Hence $\tau^{\prime}$ is almost admissible. Substitute $-\omega d_{1}$ for 
$d_{1} E$ in (111) and see that (111) is equivalent, for our choice of $d_{1}, \delta$ and $\tau^{\prime}$, to the condition

$$
\begin{aligned}
\left(d_{0}+\tau^{\prime} d_{1}\right)-\left(d_{0}+\tau^{\prime} d_{1}\right)^{*} & =\frac{\delta E}{\delta} d_{1}+\frac{\delta E^{*}}{\delta} d_{1}^{*}-\left(\frac{\delta E}{\delta}+\frac{\delta^{*} E}{\delta^{*}}\right) d_{1} \\
& =\frac{\delta E^{*}}{\delta} d_{1}^{*}-\frac{\delta^{*} E}{\delta^{*}} d_{1} .
\end{aligned}
$$

This is equivalent to the property that the element

$$
\begin{aligned}
s & =d_{0}-\left(\tau^{\prime} d_{1}\right)^{*}+\frac{1}{2}\left(\frac{\delta E}{\delta}-\frac{\delta^{*} E}{\delta^{*}}\right) d_{1}+\frac{\delta^{*} E}{\delta^{*}} d_{1} \\
& =d_{0}-\left(\tau^{\prime} d_{1}\right)^{*}+(1 / 2) \omega d_{1}=d_{0}-\left[\alpha u\left(\tau_{1} E\right)^{p}\right]^{*} \tau_{1}-(1 / 2) d_{1} E
\end{aligned}
$$

is symmetric. Clearly we can select $d_{0}$ in $\Re\left(y_{1}\right)$ so that $s$ is actually zero. This completes the proof of a final result which we state as follows.

THEOREM 8. Let $\mathfrak{D}$ be the division algebra over $\mathfrak{F}$ defined by the value $\tau=\tau^{\prime}\left(I-E^{p-1}\right)$, where

$$
\tau^{\prime}=\frac{\downarrow E}{\psi}-\frac{\psi^{*} E}{\psi^{*}}+\alpha u\left(\tau_{1} E\right) \tau_{1}^{*}
$$

for $\psi$ any element of $\mathfrak{\Re}, \alpha$ in $\mathfrak{F}$, and $\tau_{1}$ in $\Re\left(y_{1}\right)$. Then $\mathfrak{D}$ is cyclic over $\mathfrak{F}$.

The University of Chicago 